\title{
Präventive Potentiale neuer Technologien erkennen und nutzen
}

\author{
Sandra Lemanski ${ }^{1}$, Jan Vitera ${ }^{2}$, Holger Mühlan ${ }^{3}$, Silke Schmidt ${ }^{4}$ \\ ${ }^{1}$ sandra.lemanski@uni-greifswald.de \\ 2 jan.vitera@uni-greifswald.de \\ ${ }^{3}$ holger.muehlan@uni-greifswald.de \\ ${ }^{4}$ silke.schmidt@uni-greifswald.de \\ 1,2,3,4 Institut für Psychologie, Lehrstuhl Gesundheit und Prävention, \\ Universität Greifswald,, Deutschland
}

DOI: 10.14464/awic.v3i0.261

\section{ABSTRACT}

Werden im Rahmen der digitalen Transformation neue Technologien eingeführt, lassen sich seitens der Beteiligten oftmals sehr unterschiedliche Erwartungen an die Technologien und deren mögliche positive Folgen wie auch negative Auswirkungen feststellen. Ob diese dann tatsächlich eintreten oder nicht, hängt aber weniger von den eingeführten Technologien an sich ab, sondern wird maßgeblich durch die Auseinandersetzung mit zu erwartenden Effekten im Vorfeld der Einführung bestimmt. Welche Aspekte gilt es aber konkret in Betracht zu ziehen und wie können vor allem Potentiale identifiziert und genutzt werden? Diesen Fragen ist das Teilvorhaben der Universität Greifswald im Rahmen des Verbundprojektes „Gesunde Arbeit in Pionierbranchen“ (GAP) nachgegangen. Dazu wurde ein umfassendes Konzept entwickelt, welches personelle und organisationale präventive Potentiale im Kontext der Einführung neuer Technologien fokussiert. Dieses Konzept wird mittels qualitativer und quantitativer Daten in ein Manual überführt und soll vermittelt über Branchennetzwerke vor allem klein- und mittelständischen Unternehmen bei der digitalen Transformation unterstützen. Durch den offenen und modularen Aufbau des Manual-Konzeptes sind die Netzwerke in der Lage sowohl das Konzept als auch die Inhalte selbständig zu aktualisieren, zu ergänzen und zu erweitern.

Keywords: Digitale Transformation, Potentialidentifikation, Potentialnutzung, Arbeits- und Gesundheitsschutz, Fallstudien 


\section{EINFÜHRUNG}

Ausgangslage: Die Ursachen und Motive für die Einführung neuer Technologien sind so vielfältig wie die verfügbaren technologischen Möglichkeiten selbst (BMWi, 2017; Saam, Viete \& Schiel, 2016). Gemein ist aber allen, dass seitens der Beteiligten ganz verschiedene positive wie negative Erwartungen an diese Technologien geknüpft werden (BMWi, 2017; DBG, 2017). Dabei ist es nicht die Technologie als solche, welche bestimmt, ob diese Erwartungen eintreten oder nicht. Maßgeblich werden die Auswirkungen einer technologischen Neuerung davon bestimmt, wie intensiv und umfassend die unternehmensinterne Auseinandersetzung im Vorfeld gestaltet wurde, womit unter anderem klassische Aspekte des Change Managements angesprochen sind (Stolzenberg \& Heberle, 2013). Dazu gehört aber nicht nur das Abwägen und Minimieren von Risiken, sondern auch die Identifikation unternehmensspezifischer Potentiale in allen Bereichen sowie für alle Beteiligten, um die Chancen nutzen zu können. Obwohl zwar häufig von ebensolchen Chancen die Rede ist und knapp zwei Drittel der klein- oder mittelständischen Unternehmen bzw. drei Viertel der Großunternehmen die digitale Transformation als Chance bewerten (vgl. acatech, 2016), halten sich sowohl die theoretischen wie auch die praktischen Publikationen dazu eher zurück. Vielfach finden sich nur allgemeine Ausführungen oder es werden nur Teilbereiche oder bestimmte Akteure in die Betrachtungen einbezogen. Will sich ein klein- oder mittelständisches Unternehmen mit der digitalen Transformation auseinandersetzen, sind diese punktuellen Einblicke unzureichend.

Digitale Transformation und gesunde Arbeit: Von besonderer Relevanz im Kontext der digitalen Transformation sind Aspekte des Arbeits- und Gesundheitsschutzes für den sich mit Einführung neuer Technologien oder Arbeitsformen neue Herausforderungen ergeben. Die Berücksichtigung dieser Aspekte kann als Chance verstanden werden, die Arbeit von morgen gesund zu gestalten. So kann davon ausgegangen werden, dass z. B. die Einführung eines Roboters diverse Effekte auf die Beschäftigten und ihre Arbeit haben kann. Von Verschiebungen der notwendigen Kompetenzen und damit der Wertigkeit der Tätigkeiten für das Arbeitsergebnis über Monotonie durch erforderliche Überwachungsaufgaben bis hin zur Einbuße des Arbeitsplatzes sind unterschiedliche Auswirkungen denkbar. Implizit geht mit dieser Betrachtung ein enger Fokus auf die Tragweite der Veränderungen einher und nimmt nur die direkten und proximalen Effekte in den Blick. Wird der Fokus erweitert und indirekte sowie distale Auswirkungen eingeschlossen, erschließen sich neben personellen auch organisationale Potentialen, denn meist wirken sich die Veränderungen auch auf nicht direkt assoziierte Arbeitsprozesse und Strukturen innerhalb des Unternehmens sowie auf externe Faktoren (Absatzmöglichkeiten, Zielgruppen, Zusammenarbeit mit Kunden und/oder Lieferanten) aus. Die im Beispiel freiwerdenden Kapazitäten der Beschäftigten können so als Ressourcen in die Konzeption fachlicher Weiter- oder Neuqualifikation, des Einsatzes neuer Arbeitsorganisationsformen oder neuer Serviceangebote einbezogen werden. Mit Blick auf die Potentialbetrachtung und -nutzung ist dementsprechend eine ganzheitliche Betrachtung anstelle einer technikdeterministischen Analyse erforderlich. 
Zielstellung: Vor diesem Hintergrund und mit dem Ziel, gesundes Arbeiten als wesentlichen Bestandteil der digitalen Transformation zu etablieren, beschäftigt sich das Teilvorhaben der Universität Greifswald innerhalb des BMBF-geförderten Projektes "Gesunde Arbeit in Pionierbranchen“ (GAP) mit der Identifikation und Nutzung personeller und organisationaler präventiver Potentiale. Dieses Konzept mündet in einem Manual und soll netzwerkkoordiniert klein- und mittelständische Unternehmen durch die ganzheitliche Darstellung möglicher Effekte neuer Technologien auf verschiedenen organisationalen Ebenen des Unternehmens sowie verschiedene Akteure dabei unterstützen, eine frühzeitige, reflektierte sowie auf die Potentiale ausgerichtete Auseinandersetzung zu ermöglichen.

\section{VORGEHEN}

In einem ersten Schritt wurde innerhalb des GAP-Projektverbundes ein gemeinsames Referenzierungssystem entwickelt. Dieses enthält neben den Arbeitsdefinitionen zu den Begriffen der Industrie 4.0 und Digitalisierung, eine Heuristik zur Einordnung von Anwendungsfällen anhand definierter Konzepte zur digitalen Transformation. Aus diesem Grundverständnis heraus wurde ein Leitfaden erarbeitet, welcher zum einen das Fundament für die Konzeptentwicklung und gleichzeitig die Grundlage für die bereits durchgeführten und noch laufenden Fallstudien in Frontrunner-Unternehmen der digitalen Transformation bildet. Dem folgend stellen die leitfadengestützten halbstandardisierten Interviews, welche mit Vertretern des Unternehmens aus verschiedenen Bereichen und Ebenen durchgeführt und von einer Betriebsbegehung ergänzt werden, den Kern der Fallstudien dar. Aufgrund ihrer Vorreiterrolle und den bestehenden Erfahrungen können nicht nur wichtige Informationen zur Technikfolgenabschätzung gewonnen und Handlungsempfehlungen abgeleitet werden parallel ist eine fortlaufende Überprüfung und Anpassung des theoretisch entwickelten Leitfadens anhand von Praxisbeispielen möglich. Weiterhin wurden zur Konzeptentwicklung etablierte Bezugssysteme der BAuA (Joiko, Schmauder \& Wolff, 2010; Robelski, 2016; Rothe et al., 2017) und WHO (2010) sowie weitere relevante Studien (z. B. Bischoff et al., 2015) herangezogen. So kann gewährleistet werden, dass das Konzept auch „extern“ anschlussfähig ist und dementsprechend über den Projektverbund hinaus angewendet werden kann. Dieses methodische Vorgehen orientiert sich an einem bereits bewährten Vorgehen unserer Arbeitsgruppe zu interdisziplinären Analysen von psychosozialen Auswirkungen von MonitoringTechnologien (vgl. Muehlan et al., 2013; Schmidt \& Verweij, 2013). Dabei dient die initial entwickelte konzeptuelle Leitfadenstruktur als Matrix zur Integration der Informationen aus unterschiedlichen Quellen: Befunde aus einem umfassenden Literaturreview (Absicherung der „Breite“), Befunde aus den Projekt-Fallstudien (Absicherung der „Tiefe“) sowie die Ergebnisse der Mitglieder- und Beschäftigtenbefragungen (branchenbezogene Adaptation und Spezifizierung der Informationen). Im Anschluss wird diese vorläufige Version des Manuals durch Experteninterviews validiert. 


\section{KONZEPT}

Das Ergebnis des Entwicklungsprozesses lässt sich als Konzeptstruktur vereinfacht wie folgt skizzieren:

\begin{tabular}{|c|c|}
\hline $\begin{array}{l}\text { Funktionsbereich/e der neuen Technologie/n } \\
\quad \rightarrow \text { Technologie }\end{array}$ & $\begin{array}{l}\text { Da unterschiedliche Technologien vergleichbare und eine Technologie } \\
\text { verschiedene Funktionen erfüllen kann, wird in erster Linie auf die } \\
\text { Funktionsbereiche abgestellt und erst an zweiter Stelle auf eine } \\
\text { spezifische/konkrete Technologie }\end{array}$ \\
\hline Arbeitsbedingungsfaktor/en & $\begin{array}{l}\text { - Hier wurden die für die digitale Transformation } \\
\text { wesentlichen Kernaspekte einbezogen }\end{array}$ \\
\hline Moderatoren & $\begin{array}{l}\text { - Als Moderatoren werden alle förderlichen und/oder hinderlichen } \\
\text { Faktoren verstanden, welche die Auswirkungen auf individueller und } \\
\text { organisationaler Ebene beeinflussen }\end{array}$ \\
\hline Auswirkungen & $\begin{array}{l}\text { Die Auswirkungen werden weiter entsprechend der zeitlichen Folge } \\
\text { (kurz-, mittel- und langfristig) sowie in Bezug auf die betrachteten } \\
\text { Ebenen (organisational, individuell) unterschieden }\end{array}$ \\
\hline Maßnahmen & $\begin{array}{l}\text { - Bezugnehmend auf die Auswirkungen werden hier } \\
\text { Maßnahmen benannt, die zur Förderung positiver und } \\
\text { Vermeidung negativer Effekte eingesetzt werden können }\end{array}$ \\
\hline Evidenzgrad & $\begin{array}{l}\text { - In Abhängigkeit der Quellen erfolgt die Kennzeichnung des } \\
\text { Evidenzgrades (z. B. Fallstudie, Querschnittsstudie ...) }\end{array}$ \\
\hline Referenz & - Zusätzlich zur Referenz finden sich hier Verweise auf weitere Quellen \\
\hline
\end{tabular}

Abbildung 1: Struktur des Manual-Konzeptes

Quelle: $\quad$ eigene Darstellung

Die Inhalte des Manuals setzen sich, den obigen Ausführungen folgend, zum einen aus projektinternen Erkenntnissen (Fallstudien, Mitglieder- und Beschäftigtenbefragungen, Experteninterviews) und externen Quellen wie Literaturreviews, öffentlich verfügbaren Informationen (z. B. zu Gestaltungsansätzen oder Maßnahmen) zusammen und werden zum anderen in allgemeine und spezifische Inhalte unterteilt. Dabei werden die spezifischen Inhalte, wie sie beispielswiese aus den Fallstudien zur Verfügung stehen, den allgemeinen Informationen zugeordnet. So kann seitens des Nutzers dann sowohl auf sehr konkrete Inhalte im Sinne von best practice-Beispielen als auch auf allgemeine Ausführungen mit Raum zur unternehmensspezifischen Anpassung zugegriffen werden.

\section{AUSBLICK}

Das entwickelte Manual-Konzept zur Identifizierung und Nutzung personeller und organisationaler Potentiale soll netzwerkkoordiniert klein- und mittelständischen Unternehmen bei der digitalen Transformation unterstützen. Konkret wird dieses Angebot im ersten Schritt 
den Mitgliedsunternehmen der im Projekt beteiligten Netzwerke zur Verfügung gestellt. Interessierte Unternehmen können sich dann über verschiedene Einstiegsmöglichkeiten (Such- und Filterfunktionen) hinsichtlich spezifischer Fragestellungen informieren. Beabsichtig ein Unternehmen bspw. ein Assistenzsystem einzuführen, können über den Zugang „Technologie“ oder die Suchfunktion („Roboter“, „Assistenzsystem“, „Automatisierung“) die dazu verfügbaren Informationen eingesehen werden. Diese sind so aufgearbeitet, dass bezogen auf das eingangs kurz skizzierte Beispiel, eine ganzheitliche Betrachtung der zu erwartenden Auswirkungen und möglichen Maßnahmen angeregt wird. Dies wird über Hinweise, eine reflektierte Darstellung sowie über Leitfragen umgesetzt. Vor allem Unternehmen die noch am Anfang der digitalen Transformation stehen, soll das Manual den Einstieg erleichtern und praxisnahe Orientierung bieten. Aber auch für Unternehmen, die sich bereits im Prozess befinden, kann das Manual aufgrund des modularen Aufbaus sowie der vielfältigen Zugangsmöglichkeiten und Inhaltsformate wertvolle Unterstützung bietet. Die technische Umsetzung des Manuals erfolgt als Datenbank mit Exportfunktion und erlaubt einen benutzerdefinierten Zugang zu den Inhalten. Des Weiteren sind durch den modularen Aufbau des Manuals jederzeit strukturelle und inhaltliche Ergänzungen und Erweiterungen möglich.

\section{LITERATURVERZEICHNIS}

acatech (Hrsg.). (2016). Kompetenzentwicklungsstudie Industrie 4.0. München: acatech.

Bischoff, J., Taphorn, C., Wolter, D., Braun, N., Fellbaum, M., Goloverov, A., ... Scheffler, D. (2015). Studie „Erschließen der Potenziale der Anwendung von, Industrie 4.0“ im Mittelstand“. Mülheim an der Ruhr

Bundesministerium für Wirtschaft und Energie (2017). Monitoring Report Wirtschaft DIGITAL 2017. Abgerufen am 06.02.2018 unter: https://www.bmwi.de/Redaktion/DE/Publikationen/Digitale-Welt/monitoring-reportwirtschaft-digital.pdf? _blob=publicationFile $\& v=10$

DGB (2017). DGB-Index Gute Arbeit. Verbreitung, Folgen und Gestaltungsaspekte der Digitalisierung in der Arbeitswelt. Auswertungsbericht auf Basis des DGB-Index gute Arbeit 2016. Berlin: Institut DGB-Index Gute Arbeit

Joiko, K., Schmauder, M. \& Wolff, G. (2010). Psychische Belastung und Beanspruchung im Berufsleben. Erkennen - Gestalten (5. Aufl.). Dortmund: Bundesanstalt für Arbeitsschutz und Arbeitsmedizin

Muehlan, H., Rhode, D. \& Schmidt, S. (2013). Investigating the mental and social impact of personal health monitoring. In Schmidt, S. \& Rienhoff, O. (Eds.), Interdisciplinary Assessment of Personal Health Monitoring (pp. 95-103). Amsterdam: IOS Press

Robelski, S. (2016). Psychische Gesundheit in der Arbeitswelt - Mensch-Maschine-Interaktion. Dortmund: Bundesanstalt für Arbeitsschutz und Arbeitsmedizin. DOI: 10.21934/baua:bericht20160713/4d

Rothe, I., Adolph, L., Beermann, B., Schütte, M., Windel, A., Grewer, A., Lenhardt, U., Michel, J., Thomson, B. \& Formazin, M. (2017). Psychische Gesundheit in der Arbeitswelt - 
Wissenschaftliche Standortbestimmung. Dortmund: Bundesanstalt für Arbeitsschutz und Arbeitsmedizin. DOI: 10.21934/baua:bericht20170421

Saam, M., Viete, S. \& Schiel, S. (2016). Digitalisierung im Mittelstand: Status Quo, aktuelle Entwicklungen und Herausforderungen. Forschungsprojekt im Auftrag der KfW Bankengruppe. Mannheim: Zentrum für Europäische Wirtschaftsforschung GmbH

Schmidt, S. \& Verweij, M. (2013). The PHM Methodology. Interdisciplinary Technology Assessment of Personal Health Monitoring. In S. Schmidt \& O. Rienhoff (Eds.), Interdisciplinary Assessment of Personal Health Monitoring (pp. 13-20). Amsterdam: IOS Press

Stolzenberg, K. \& Heberle, K. (2013). Change Management. Veränderungsprozesse erforlgreich gestalten - Mitarbeiter mobilisieren. Vision, Kommunikaiton, Beteiligung, Qualifizierung (3. überarbeitet Aufl.). Berlin: Springer-Verlag

World Health Organization (2010). WHO Healthy Workplace Framework and Model: Background and Supporting Literature and Practice. WHO: Geneva 